\title{
Lengorgement des départements d'urgence et le blocage d'accès
}

\author{
Andrew Affleck, MD*; Paul Parks, MD; Alan Drummond, $\mathrm{MD}^{\ddagger}$; Brian H. Rowe, MD, MSc ${ }^{\S}$; \\ Howard J. Ovens, MD"
}

\section{SOMMAIRE}

L'engorgement des départements d'urgence se définit comme une situation dans laquelle la demande de services d'urgence excède la capacité d'un département à offrir des soins de qualité dans des délais acceptables. ${ }^{1,2}$ L'engorgement des départements d'urgence est un enjeu clé de la médecine d'urgence au Canada depuis plus de 20 ans. En dépit de la sensibilisation accrue au plan politique, administratif et public, les situations d'engorgement des départements d'urgence ne cessent d'accroître en fréquence et en gravité. ${ }^{3}$ Patients souffrants, délais d'attente prolongés, niveaux de service en détérioration, résultats indésirables pour les patients et la capacité de retenir du personnel d'expérience dans les services d'urgence sont tous des incidences négatives de ce problème continu.

Contrairement aux perceptions du public, l'engorgement des départements d'urgence n'est pas occasionné par un usage inadéquat des services d'urgence ou par un nombre élevé de cas moins graves se présentant au département d'urgence (DU). L'incapacité des patients admis aux services d'urgence d'avoir accès à des lits destinés aux patients hospitalisés est le facteur le plus important causant l'engorgement des départements d'urgence dans les hôpitaux canadiens.

Malgré l'importance de cette situation, il n'existe aucune norme nationale à l'heure actuelle visant à déterminer la gravité du problème (et par conséquent, l'identification des facteurs occasionnant des résultats négatifs). Par cet énoncé de position, l'ACMU proposera des recommandations de normes nationales (cibles) de rendement pour les DU dans le but d'aider à mettre fin à ce problème. Les suggestions de cibles sont comme suit:

i. Durée pour l'évaluation initiale du médecin (EIM):

- Médiane de 1 heure, $90^{\mathrm{e}}$ percentile de 3 heures. ii. Durée pour le transfert vers un lit d'bôpital:

- Médiane de 2 heures, $90^{\mathrm{e}}$ percentile de 8 heures. iii. $D D S D U$ :

- ÉTG IV/V patients sortants - Médiane de 2 heures, $90^{\mathrm{e}}$ percentile de 4 heures;

- ÉTG I-III patients sortants - Médiane de 4 heures, $90^{\mathrm{e}}$ percentile de 8 heures;

- Patients hospitalisés (tous les niveaux de l'ÉTG) - Médiane de 8 heures, $90^{\mathrm{e}}$ percentile de 12 heures.

Nous croyons que l'adoption de normes nationales (voir les recommandations pour plus de détails) procurera des objectifs à réaliser pour chaque province ou territoire en créant un mécanisme de comparaison de leur progrès avec ceux de leurs collègues. Nous comprenons que selon leurs circonstances et leur situation actuelle, certains hôpitaux trouveront ces cibles difficiles à atteindre, tandis que d'autres réalisent ou surpassent peut-être déjà ces objectifs. Nous croyons que tout le monde bénéficiera d'un ensemble de mesures et de normes communes.

Tiré de: *Centre régional des sciences de la santé de Thunder Bay, École de médecine du Nord de l'Ontario, Thunder Bay, ON; Coprésident des affaires publiques de I'ACMU; ${ }^{\dagger}$ Hôpital régional de Medicine Hat, Medicine Hat, AB; ${ }^{\ddagger}$ Hôpital du district de Perth et Smith Falls, Perth, ON; Coprésident des affaires publiques de I'ACMU; ${ }^{5}$ Département de médecine d'urgence et école de la santé publique, Université de I'Alberta, Edmonton, AB; et "Centre d'urgence Schwartz/Reisman, hôpital Mount Sinai et Collège des médecins de famille, Université de Toronto, Toronto, ON.

Correspondance à: Dr. Howard Ovens, Centre d'urgence Schwartz/Reisman, hôpital Mount Sinai, Toronto, ON M5G 1X5; howard.ovens@utoronto.ca. 
L'engorgement des départements d'urgence est une préoccupation de santé publique dont l'origine s'étend bien au-delà des murs des DU canadiens. Cette situation reflète le besoin pour des solutions et des interventions à plusieurs niveaux au sein du système de santé. Les solutions citées dans cet énoncé de position reflètent ce besoin, tout en ne minimisant pas le facteur le plus important responsable de l'engorgement des départements d'urgences: les délais pour les patients admis au DU d'avoir accès à des lits d'hôpitaux.

\section{POSITION DE L'ACMU}

1. Le principal problème occasionné par l'engorgement des départements d'urgence est un blocage de la prestation des soins de santé requis pour les patients se présentant au DU dans un délai et un endroit raisonnables. Ceci engendre un accès réduit aux soins de santé ou «blocage d'accès» (BA). ${ }^{*} \mathrm{Le}$ blocage d'accès est souvent occasionné par des problèmes de capacité et d'efficacité du système provenant à l'extérieur du DU.

L'engorgement des départements d'urgence est associé à une hausse du taux de mortalité et de résultats négatifs chez les patients examinés dans des DU engorgés, que ce soit pour être admis ou pour obtenir leur congé. 4.5 Ce sujet a été étudié en profondeur et peut être conceptualisé au moyen du modèle de l'afflux, de l'intervention et de l'issue, où une approche exhaustive et juridictionnelle est requise pour traiter les facteurs ayant un impact sur le flux des patients à l'extérieur des services d'urgence; dans la communauté, dans le reste de l'hôpital de soins actifs et dans le secteur des soins continus pour les patients en phase post-aiguë. Un résumé des résultats sur les interventions et les stratégies visant à réduire l'engorgement a été publié. ${ }^{6,7}$ Des approches globales pour répondre

\footnotetext{
*Pour des raisons de cohérence, l'expression «engorgement des départements d'urgence" est utilisé dans ce document pour désigner spécifiquement les situations de blocage d'accès dans les DU. La transition vers l'usage du terme "blocage d'accès" a récemment été encouragée afin de renforcer le concept et pour faire comprendre que I'engorgement des départements d'urgence est une forme de blocage d'accès et dont l'origine et les causes proviennent généralement Á I'extérieur du DU. Par souci de cohérence, nous avons choisi de continuer Á utiliser l'expression "engorgement des départements d'urgence" pour décrire les situations de blocage $d^{\prime}$ accès qui surviennent au sein du DU.
}

aux problèmes d'engorgement des départements d'urgence d'un point de vue systémique devraient comprendre:

a. Un accès transparent et facile à des données valides et fiables pour évaluer le rendement des hôpitaux ${ }^{8}$ au moyen de définitions nationales normalisées selon l'Échelle canadienne de triage et de gravité pour les départements d'urgence (ÉTG), le Système d'information sur les départements d'urgence canadiens (SIDUC), les groupes de travail nationaux et la base de données du Système national d'information sur les soins ambulatoires (SNISA);

b. La mise en place de cibles de rendement et de normes pour fixer des délais clés relatifs au DU et à l'hospitalisation;

c. L'élaboration de rapports publics en temps opportun portant sur les cibles de rendement et la réussite des critères établis;

d. Des incitations financières (initiatives de paiement à la performance) devraient être évaluées par les hôpitaux et les fournisseurs de soins de la santé afin d'accroître leur rendement;

e. De la formation et de l'éducation pour les hôpitaux sur les meilleures pratiques pour améliorer les processus relatifs au roulement des patients au sien du DU à l'aide de ressources fondées sur des données probantes;

f. Une sensibilisation à l'accès communautaire pour des soins de longue durée doit devenir une priorité locale, provinciale et nationale;

g. Une sensibilisation:

i. À la capacité des soins de courte durée (cible maximale au-dessous des taux d'occupation de $95 \%$ );

ii. Aux autres niveaux de soins (ANS) dans des environnements de soins de courte durée (cible maximale de 5\% des taux d'occupation);

iii. À la capacité adéquate de soins de longue durée (SLD) et de soins en phase post-aiguë;

iv. Pour un soutien communautaire et à domicile pour des groupes vulnérables, tel que les personnes âgées fragiles.

2. L'usage d'intervalles normalisés pour le suivi du rendement des hôpitaux et l'élaboration de rapports destinés au public sont importants pour permettre des comparaisons de rendement inter-juridictionnelles. Avec l'heure de départ étant l'heure de 
l'enregistrement ou du triage, les intervalles ou les paramètres de rendement devraient comprendre:

a. «Délais d'attente» - Les intervalles où le patient ne fait qu'attendre:

i. Le délai d'évaluation initiale par le médecin - et la durée totale entre l'enregistrement initial/le triage et l'évaluation par un médecin;

ii. Le délai du transfert des patients des services médicaux d'urgence (SMU): «temps nécessaire pour décharger le patient de l'ambulance» - la durée entre l'arrivée du patient et sa prise en charge par le DU;

iii. Le délai de consultation: idéalement, le délai entre la demande de consultation d'un patient et l'arrivée du médecin;

iv. Le délai de transfert à des lits d'hôpitaux pour les patients hospitalisés: le délai entre la décision d'hospitalisation et le transfert/ départ actuel vers l'hôpital.

b. «Délais des soins» - Les intervalles comprenant à la fois les soins et l'attente:

i. La durée totale du séjour dans le département d'urgence (durée du séjour dans le DU ou DDS DU);

ii. Le délai entre l'arrivée et les demandes de consultation (pour les patients recevant une consultation, ceci comprend le temps de traitement du médecin d'urgence et souvent le temps pour qu'une imagerie diagnostique soit réalisée - et un rapport élaboré - et les délais d'exécution des laboratoires);

iii. Le délai entre la demande de consultation et la décision quant aux dispositions à prendre (pour les patients recevant une consultation, ceci est le temps de traitement du consultant).

3. Le format pour l'élaboration de rapports destinés au public est fondamental. Les principes clés comprennent:

a. Populations distinctes: Différencier les patients nécessitant une hospitalisation et ceux pouvant recevoir leur congé du département d'urgence en toute sécurité.

b. Rapports de données non agrégées: L'engorgement des départements d'urgence survient principalement dans les hôpitauxuniversitaires etleshôpitauxurbainsàvolumealevé La compilation de données régionales ou provinciales atténuera considérablement les problemes locaux. Des rapports présentantle rendement des installations individuelles doiventêtre daborés séparément.

c. Format des paramètres: Pour le suivi du rendement interne, les rapports de paramètres situés au $90^{\mathrm{e}}$ percentile ont des avantages et ils sont recommandés pour les professionnels des soins de santé et les administrateurs du système. D'un autre côté, l'élaboration de rapports destinés au public nécessite l'usage de médianes qui sont mieux comprises par le public et les patients. Les moyennes présentent des problèmes de données croisées et devraient être évitées.

4. Les cibles sont une composante importante pour l'amélioration du rendement. Il existe très peu de données pour guider l'établissement de cibles pour les délais d'attente dans les DU, mais les cibles devraient être déterminées au moyen des meilleures données existantes conjointement avec un consensus d'experts. Idéalement, les cibles devraient être harmonisées à travers toutes les juridictions afin de permettre une comparaison du rendement. Sans mesures objectives et des critères d'accès au système, il peut s'avérer difficile de quantifier le niveau de blocage d'accès au sein d'un hôpital ou d'une province. Pire encore, sans évaluer le succès de la réalisation des objectifs au fil du temps, il peut être très difficile d'évaluer si les ajustements d'un système conçu pour diminuer l'engorgement ont l'effet désiré. Au minimum, il est recommandé que des cibles soient établies pour les paramètres suivants, et qu'elles soient basées sur des objectifs provinciaux existants et un consensus d'experts. Les cibles suggérées sont les suivantes:

i. Durée pour l'évaluation initiale du médecin (EIM):

- Médiane de 1 heure, $90^{\mathrm{e}}$ percentile de 3 heures. ii. Durée pour le transfert vers un lit d'hôpital:

- Médiane de 2 heures, $90^{\mathrm{e}}$ percentile de 8 heures iii. DDS DU:

- ÉTG IV/V patients sortants

- Médiane de 2 heures, $90^{\mathrm{e}}$ percentile de 4 heures; 


\section{- ÉTG I-III patients sortants}

- Médiane de 4 heures, $90^{\mathrm{e}}$ percentile de 8 heures;

- Patients hospitalisés (tous les niveaux de l'ÉTG)

- Médiane de 8 heures, $90^{\mathrm{e}}$ percentile de 12 heures.

5. Il est important de garder à l'esprit qu'il y a une différence considérable entre les «délais d'attente» et «la durée du séjour». Les délais d'attente sont les intervalles où un patient attend quelque chose (p. ex: les soins d'un médecin ou d'être assigné à un lit). Les marqueurs de la durée du séjour mesurent le temps écoulé pour qu'un patient reçoive des soins, incluant le temps d'évaluation et de traitement. Bien que l'expérience d'attendre et de recevoir des soins peuvent être étroitement liés lors d'une visite au DU, ils sont souvent confondus. Il est important de fournir des données précises lors de publications et de discussions de ces chiffres, car le fait de passer un total de 8 heures dans un DU, incluant l'évaluation, les diagnostiques complexes et le traitement (DDS DU) est considérablement différent que d'attendre 8 heures dans une salle d'attente à attendre d'être examiné par un médecin (durée pour l'EIM).

\section{INTRODUCTION}

L'engorgement des DU est un problème multidimensionnel et complexe qui est conceptualisé au moyen du modèle de l'afflux, de l'intervention et de l'issue. ${ }^{9-11}$ Bien que l'attention médiatique souligne des facteurs relatifs à l'afflux et l'usage inadéquat de DU à travers le Canada, la cause principale et définitive de l'engorgement du DU est l'engorgement des hôpitaux (également connu sous le nom de «blocage d'accès».11 L'engorgement des hôpitaux peut également être conceptualisé par le même modèle: afflux (p. ex: les admissions non-urgentes et l'hospitalisation dans les DU, l'intervention (p. ex.: les services aux patients et le roulement) et l'issue (p. ex.: le congé d'hôpital, les ressources de soins communautaires, l'accès aux SLD).

\section{BACKGROUND}

L'ACMU a publié son premier énoncé de position sur l'engorgement des départements d'urgence en 2001, avec une révision en 2009. Le premier document identifiait et définissait le problème de l'engorgement des départements d'urgence, ce qui a aidé à proposer des temps d'attente dans les services d'urgence sur des forums provinciaux, en plus de faire en sorte que les temps d'attentes dans DU soient inclus dans la liste nationale des temps d'attente émise par l'Association médicale canadienne (AMC). Ayant identifié l'engorgement des départements d'urgence comme une préoccupation grandissante du système de santé, le deuxième document soulignait les origines de l'engorgement des départements d'urgence sur l'ensemble du système et recommandait des cibles de temps d'attente pour améliorer les soins prodigués aux patients, en plus de mettre l'accent sur le fait que des solutions pour contrer le blocage d'accès devaient être trouvées pour l'ensemble du système. Depuis le dernier énoncé de position en 2009, des changements sont survenus rapidement au Canada et au plan international. Au niveau provincial, plusieurs provinces ont pris des mesures pour observer et trouver des solutions au temps d'attente dans les DU. Au fil du temps, il y a eu une appréciation grandissante pour les causes multifactorielles de l'engorgement des départements d'urgence et une approche sur l'ensemble du système pour régler le problème de blocage d'accès est maintenant généralement accepté.

Les principaux facteurs responsables des longs temps d'attente dans les DU et de l'engorgement des départements d'urgence s'infiltrent dans presque tous les niveaux du système de santé canadien. Par conséquent, on a assisté au passage d'une approche orientée uniquement sur l'engorgement et les processus observés au sein du DU à une approche qui se concentre sur les facteurs causatifs réels de l'engorgement des départements d'urgence et du blocage d'accès qu'éprouvent les patients, et ce, à de multiples niveaux.

Du côté de l'afflux, des changements au niveau des soins primaires ont également causé un blocage d'accès pouvant contribuer à l'engorgement des départements d'urgence. Les patients sans fournisseur de soins primaires (FSP) peuvent se tourner aux services d'urgence comme leur seul recours à des soins de santé, tandis que les patients qui ont un FSP, mais qui ne peut répondre aux demandes semi-urgentes, se tournent également vers les urgences pour des soins rapides. Ceci engendre une situation où plus de patients ont recours au DU, et pire encore, une grande proportion de ces patients ont manqué des occasions pour recevoir des soins préventifs et se présentent avec des maladies qui n'ont fait qu'empirer avec le temps. De plus, l'âge 
des patients se présentant au DU et la complexité de leurs problèmes ont augmenté. Par conséquent, dans plusieurs DU, il y a un plus grand besoin d'examens approfondis, d'imageries médicales et de consultations avancées, rallongeant davantage la durée de l'hospitalisation des patients et contribuant ainsi à l'engorgement. Enfin, l'utilisation des DU pour prodiguer des soins épisodiques et traiter des conditions chroniques créée également un plus grand besoin pour une meilleure communication entre les DU et les FSP afin de mieux coordonner l'ensemble des soins pour les patients.

En matière d'intervention, les patients nécessitant un ANS peuvent avoir un impact considérable sur l'engorgement des départements d'urgence en occupant des lits de soins de courte durée pouvant servir à des patients du DU récemment hospitalisés. En plus de la capacité réduite de lits occasionnée par des niveaux élevés de patients exigeants un ANS, les patients en attente d'un placement pour un ANS ne reçoivent pas les soins adéquats dans un endroit optimal, ce qui peut avoir une répercussion sur leurs résultats et leur expérience. Leurs besoins doivent donc être pris en compte dans la recherche d'une solution au problème de l'engorgement des départements d'urgence.

Cet énoncé de position servira à mettre à jour les énoncés précédents afin de refléter ces changements. L'objectif de cette mise à jour est d'ajouter des expériences récentes et de la documentation scientifique à la discussion dans l'espoir de créer un document qui pourra être utilisé pour résoudre les multiples causes de l'engorgement des départements d'urgence.

\section{DÉFINITION DU PROBLÈME}

Les délais en soins d'urgence peuvent survenir à plusieurs niveaux. Tel que soulignée dans le sommaire, l'incapacité des patients admis aux services d'urgence d'avoir accès à des lits destinés aux patients hospitalisés est le principal facteur responsable de l'engorgement des départements d'urgence dans les hôpitaux canadiens les plus achalandés. Bien que les pressions d'afflux dans les DU peuvent contribuer à l'engorgement des départements d'urgence dans certaines communautés, surtout lorsqu'il est difficile pour les patients d'avoir accès à un FSP, la grande majorité du temps les goulets d'étranglement sont situés «en aval»du DU et peuvent survenir du côté de l'issue du roulement des patients. Les problèmes associés avec le roulement des patients admis au DU pour être ensuite transférés vers l'hôpital et éventuellement obtenir leur congé et retourner dans la communauté peuvent être causés par plusieurs facteurs. À de différents moments, dans des hôpitaux ou des communautés différentes, les problèmes peuvent être basés sur des limitations de capacité et d'efficacité et peuvent comprendre:

- Une utilisation sous-optimale des lits d'hôpitaux pour des soins de courte durée, incluant l'accès à des diagnostiques;

- Une pénurie de capacité de lits d'hôpitaux pour des soins de courte durée - le nombre de lits actuels peut être inadéquat ou les lits peuvent être bloqués pour des raisons de budget ou pour d'autres raisons incluant la présence de patients nécessitant un ANS;

- Une pénurie de personnel au DU (incluant les médecins);

- Les médecins/consultants et les programmes offrants des services aux patients hospitalisés;

- Des ressources limitées pour des soins communautaires - à la fois les ressources pour les soins à domiciles et les soins pour les patients en phase post-aiguë, tels que les soins à long terme et les services de réhabilitation;

- Le manque d'intégration des ressources communautaires et hospitalières;

- Une mauvaise communication entre les installations de soins de courte durée et les FSP lorsque les patients sont prêts à obtenir leur congé, mais qu'ils nécessitent un suivi;

- La confusion à savoir qui est responsable du patient aux divers stades des soins.

Avec les pénuries de lits d'hôpitaux et les problèmes récurrents relatifs à la capacité de prodiguer des soins de courte durée, les hôpitaux sont de plus en plus confrontés à des situations où il y a plus de patients malades que les lits d'hôpitaux pour les recevoir. L'approche actuelle pour régler le problème du blocage d'accès en raison de l'engorgement des hôpitaux implique le retardement du flux sortant de patients admis au DU vers des endroits appropriés pour des patients hospitalisés. Cette pratique «d'entreposage» des patients donne lieu à un usage excessif et inadéquat des DU. «L'hébergement» de patients hospitalisés au sein du DU engendre un engorgement des départements d'urgence et entraîne des délais prolongés pour l'examen de nouveaux patients se présentant au DU.

Des sondages ont démontré que les patients tentent plusieurs autres options avant de se tourner au DU. ${ }^{12}$ Or, les patients présentant des cas moins graves et 
moins urgents n'occupent pas des civières destinées aux soins urgents, ils nécessitent peu de soins infirmiers et ils requièrent généralement des traitements de courte durée. Le mythe de «l'usage inadéquat» des DU devrait être éliminé entièrement, et les administrateurs et les politiciens devraient être encouragés à ne pas attribuer l'engorgement des départements d'urgence au manque d'accès à services de santé d'urgence pour les patients ambulatoires. Bien que les patients ayant obtenu leur congé d'hôpital ne soient pas la cause de l'engorgement du DU, des améliorations au processus hospitalier pour ce groupe diminueraient leurs temps d'attente et amélioreraient leur expérience. Tous les DU canadiens devraient s'engager à continuellement améliorer la qualité de leurs services afin de s'assurer qu'ils maintiennent les meilleures pratiques et optimisent les ressources du DU et par le fait même, l'expérience des patients. L'amélioration et l'optimisation des soins prodigués au sein des DU devraient être une priorité pour tous les hôpitaux. Toutefois, ce processus d'optimisation ne réglera pas les goulets d'étranglement situés en aval de l'issue et qui sont la cause réelle de l'engorgement des départements d'urgence.

En raison du problème quasi universel et récurrent des limitations des lits d'hôpitaux au Canada, l'engorgement des départements d'urgence est une conséquence directe de l'engorgement des hôpitaux, qui à son tour contribue principalement au blocage d'accès. ${ }^{13}$ Au Canada, le problème de l'engorgement des départements d'urgence est le plus critique dans les DU de traumatologie, de soins tertiaires, les DU universitaires et dans les hôpitaux à grand volume. ${ }^{3}$ Les conséquences de l'engorgement des départements d'urgence sont toutefois semblables sur l'ensemble du système de soins d'urgence; les hôpitaux d'attache et les ambulances sont incapables d'accéder à des centres de soins d'urgences secondaires et tertiaires dans un délai raisonnable. Par exemple, malgré le fait qu'ils ont la capacité d'offrir des soins de courte durée adéquats au plan local, certains hôpitaux sont souvent confrontés au blocage d'accès sous la forme d'un délai de transfert à des soins de longue durée pour leurs patients. Cette forme de blocage d'accès est un problème important pour les médecins et les patients de régions rurales lorsque les médecins sont incapables de transférer des patients nécessitant des soins de niveau plus élevé à des centres urbains qui sont fréquemment débordés.

Des pressions sur les services ambulanciers peuvent survenir lorsque les DU sont engorgés de patients hospitalisés et que les ambulanciers sont incapables de transférer les patients au personnel du service d'urgence en temps opportun. Les délais de déchargement des ambulances ou dans de rares cas, le détournement des ambulances, sont tous les deux des exemples de blocage d'accès où l'engorgement des départements d'urgence impacte et retarde l'accès à des soins pré-hospitaliers. Bien que l'engorgement des départements d'urgence peut compromettre les soins pour les patients des SPU en attente d'être transféré vers un lieu de soins du DU, il peut également mener à des pressions sur les employés des SPU et occasionner de plus longs temps de réponse pour des nouveaux appels. Cela compromet la sécurité des patients en situation d'urgence dans la communauté alors que le blocage d'accès se déplace en amont.

Le blocage d'accès peut également survenir à de multiples niveaux au sein des hôpitaux. Dans le DU, lorsque les patients hospitalisés occupent des civières du DU pour une période de temps prolongée, ils bloquent l'accès à ces espaces de soins à des patients malades ou blessés dans la salle d'attente et augmentent les temps d'attente pour les patients nouvellement arrivés. Pour les patients hospitalisés hébergés dans le DU, les soins prodigués n'équivalent pas les soins offerts dans l'hôpital et il y a donc un blocage d'accès à des soins adéquats pour les patients hospitalisés. Dans de nombreux hôpitaux canadiens, des chirurgies non urgentes ont été retardées ou annulées dans un effort pour contrer l'engorgement des DU et des hôpitaux. Par conséquent, les patients en attente de chirurgies prévues sont confrontés au blocage d'accès. Dans les départements d'hôpitaux, alors que les taux d'engorgement des hôpitaux s'accroissent, les charges de travail des services infirmiers sont souvent perçues comme étant dangereuses et la satisfaction des médecins/ patients décroît lorsque les protocoles de surcapacité (PS) sont instaurés.

En 2009, le Canada n'avait que 1,7 lit d'hôpital destiné aux soins de courte durée pour 1000 Canadiens et se classait $33^{\mathrm{e}}$ sur 34 pays membres de l'Organisation de coopération et de développement économique (OCDE) (la moyenne de l'OCDE était de 3,4/1000).14 La pénurie de lits d'hôpitaux pour les soins de courte durée au Canada signifie que la majorité des hôpitaux fonctionnent couramment avec des taux d'occupation insoutenables de plus de 95\%, un niveau rendant inévitables les pénuries régulières de lits, les crises périodiques de lits et les engorgements des hôpitaux. ${ }^{15-17}$ 
OEuvrer à des capacités supérieures à $95 \%$ du taux d'occupation ne permet aucune flexibilité du système pour accommoder les périodes de pointes naturelles dans les volumes de patients et des hospitalisations qui surviennent sur une base périodique.

La capacité d'offrir des lits d'hôpitaux pour des soins de courte durée peut également être affectée de manière considérable par des patients qui occupent des lits destinés à des soins de courte durée, mais qui nécessitent actuellement «d'autres niveaux de soins» (p. ex.: soins de longue durée, réhabilitation, etc.), mais qui ne peuvent $\mathrm{y}$ avoir accès en raison de pénuries dans les ressources communautaires et les capacités de lits pour les patients en phase post-aiguë. Ces patients occupent jusqu'à $20 \%$ du taux d'occupation des lits d'hôpitaux pour les soins de courte durée, et par conséquent, ils contribuent à l'engorgement des DU et au blocage d'accès en empêchant les patients du DU d'avoir accès à des lits d'hôpitaux. ${ }^{18}$ La majorité des patients nécessitant des ANS sont des personnes âgées. Avec l'allongement de l'espérance de vie et le vieillissement de la population, ces problèmes ne feront qu'empirer s'ils ne sont pas résolus.

Comme le démontrent les exemples ci-dessus, le problème de blocage d'accès en général, et plus précisément, la préoccupation grandissante de l'engorgement des départements d'urgence, sont des enjeux à facettes multiples et une intervention singulière ne peut être efficace. Toute tentative visant à résoudre l'engorgement des départements d'urgence nécessitera une approche sur l'étendue du système qui devra tenir compte des facteurs relatifs à l'afflux (un meilleur accès à des soins de base et des soins continus améliorés pour les patients souffrants de conditions chroniques), aux enjeux d'intervention et de l'optimisation du DU, en plus de traiter les goulets d'étranglement de l'issue et le roulement des patients hospitalisés (de la capacité des soins de courte durée et l'amélioration de l'efficacité jusqu'à la capacité des soins communautaires et les soins pour les patients en phase post-aiguë).

\section{RECOMMANDATIONS}

Les recommandations suivantes ont été élaborées grâce à des documents fondés sur des données probantes et les opinions et le consensus d'experts de l'ACMU.

i. Mettre sur pied des normes nationales pour l'établissement d'intervalles clés dans les DU et l'élaboration de rapports publics:

L'ACMU recommande la mise sur pied de normes nationales pour l'établissement d'intervalles clés pour les patients recevant des soins au DU. Afin d'encourager la transparence et pour s'assurer que cet enjeu demeure au premier plan dans l'attention du public, ces cibles et les mesures de rendement non-agrégées des hôpitaux individuels devraient être annoncés publiquement. Toutes les normes doivent être mesurables et liées à un cadre de responsabilisation dans l'objectif d'évaluer adéquatement le rendement. Des données fiables, complètes et précises doivent également être recueillies dans chaque DU pour mesurer le progrès et évaluer les interventions.

En ce qui concerne l'élaboration de rapports destinés au public, la médiane est la donnée la mieux comprise par la population et elle reflète l'expérience typique d'un patient. Les cibles au $90^{\mathrm{e}}$ percentile devraient également être mesurés et rapportés puisqu'elles reflètent mieux la majorité des expériences et sont un meilleur outil pour identifier les goulets d'étranglement existants et pour juger l'amélioration des processus. Elles peuvent également servir d'incitation, comme les initiatives de paiement à la performance.

Table 1. Variation in Emergency Department wait-time targets (as of November 2011)

\begin{tabular}{|c|c|c|c|}
\hline & Admits & High Acuity Discharges & Low acuity discharges \\
\hline Nova Scotia & 8 hours $90^{\text {th }} \%$-ile & 8 hours $90^{\text {th }} \%$-ile & 4 hours 90 th $\%$-ile \\
\hline Quebec & 12 hour (mean) & \multicolumn{2}{|c|}{8 hours (mean) ${ }^{*}$ applies only to stretcher patients. } \\
\hline Ontario & 8 hours $90^{\text {th }} \%$-ile & 8 hours $90^{\text {th }} \%$-ile & 4 hours $90^{\text {th }} \%$-ile \\
\hline Manitoba & & N/A & \\
\hline Saskatchewan & & $\mathrm{N} / \mathrm{A}$ & \\
\hline Alberta & 8 hours $90^{\text {th }} \%$-ile & \multicolumn{2}{|c|}{4 hours $90^{\text {th }} \%$-ile } \\
\hline British Columbia & 10 hours $75^{\text {th }} \%$-ile & 4 hours $75^{\text {th }} \%$-ile & 2 hours $75^{\text {th }} \%$-ile \\
\hline
\end{tabular}


Il est prévu que les hôpitaux à travers le pays seront initialement à des niveaux variant de rendement, mais les patients peuvent s'attendre à ce que nous travaillions vers une norme commune en matière de services. En général, il est raisonnable de s'attendre à une amélioration de 5 à $10 \%$ par année pour atteindre ces cibles.

À l'heure actuelle, il existe de nombreuses cibles différentes en place au Canada - voir le Tableau 1 l'ACMU incite les provinces à se réunir et à définir des cibles communes et des normes pour l'élaboration de rapports afin que les Canadiens puissent savoir comment leur communauté se compare à d'autres à travers le pays.

1. Durée pour l'EIM: Ceci est l'intervalle entre le triage ou l'enregistrement et l'examen du patient par un médecin. La majorité des patients définiraient intuitivement cet intervalle comme leur «temps d'attente» lors d'une visite à l'urgence et cet intervalle correspond aux taux de «départs sans examen», à la satisfaction globale des patients et à la durée totale du séjour au DU.

L'ACMU recommande une cible d'une heure à la médiane et de trois heures au $90^{\mathrm{e}}$ percentile.

2. Durée pour le transfert vers un lit d'hôpital: Ceci est l'intervalle entre la décision d'hospitaliser un patient et son départ vers l'hôpital. Il s'agit de l'autre intervalle d'attente clé et reflète la disponibilité de lits au moment de l'admission, en plus de l'efficacité de l'administration hospitalière à assigner des lits et à arranger le transfert des soins et du transport. Les patients admis attendent dans le DU dans des circonstances inconfortables pendant de longues périodes de temps et cette situation devrait être évitée dans un système de santé au fonctionnement et aux ressources optimales.

L'ACMU recommande une cible de deux heures à la médiane et de 8 heures au $90^{\mathrm{e}}$ percentile.

3. Durée totale du séjour dans le DU (DDS DU): Ceci est la durée entre l'arrivée au triage ou à l'enregistrement et le départ vers la maison ou vers l'hôpital. Ceci reflète l'ensemble de l'expérience du patient, incluant les soins et l'attente. Dans certains cas, un meilleur soin nécessitera un séjour plus long, ce qui est reflété en partie dans les cibles de temps variantes selon la gravité et les dispositions. a. Patients sortants à faible gravité (ÉTG IV ou V à l'arrivée): L'AMCU recommande une cible de 2 heures à la médiane et de 4 heures au $90^{\mathrm{e}}$ percentile;

b. Patients sortants à haute gravité (ÉTG I-III à l'arrivée): L'ACMU recommande une cible de 4 heures à la médiane et de 8 heures au $90^{\mathrm{e}}$ percentile;

c. Les patients hospitalisés: L'ACMU recommande une cible de 8 heures à la médiane et de 12 heurs au $90^{\mathrm{e}}$ percentile.

ii. Concilier les normes relatives à la durée du séjour dans le DU (DDS DU) à des incitations et des investissements dans l'infrastructure: Les normes relatives à la DDS DU doivent être associées à des incitations et à des investissements dans les infrastructures pour qu'un changement significatif soit réalisé. Le Royaume-Uni et l'Ontario ont réussi à réaliser des baisses considérables dans les temps d'attentes au DU suivant l'adoption de cibles pour la DDS DU sur l'ensemble de leurs juridictions. ${ }^{19}$ Ceci était couplé d'incitations financières, de mesures de reddition de comptes et d'efforts pour contrer les délais d'accès à des lits d'hôpitaux, des médecins spécialistes et des diagnostics.

iii. Rendre obligatoire un répertoire national de données portant sur les visites au DU: C'est une énigme nationale que les données des visites au DU ne sont pas toutes tenues et rapportées à une source centrale. Seuls l'Alberta et l'Ontario contribuent toutes leurs données relatives aux visites au DU à la base de données du Système national d'information sur les soins ambulatoires (SNISA) maintenue par l'Institut canadien d'information sur la santé (ICIS). Un accès transparent et facile à des données valides et fiables pour mesurer le rendement à l'aide des définitions nationales normalisées conformément à celles proposées par les groupes de travail nationaux de l'ACMU, de l'ÉTG et le SIDUC devrait être une priorité provinciale et fédérale.

iv. Optimiser la gestion des lits et planifier de manière proactive de la capacité de lits: En plus d'accroître le nombre absolu de lits pour des soins de courte durée, la capacité de lits pour les patients hospitalisés peut également être améliorée en optimisant la gestion des lits. Des stratégies de gestion efficaces en ce qui trait des lits d'hôpitaux 
devraient atténuer le niveau de variabilité des admissions et des congés. Afin de réaliser une meilleure gestion, une attention particulière devrait être portée sur les secteurs suivants: la planification des congés, l'atténuation de la fluctuation des chirurgies, les procédures d'admission, la planification de la capacité, la planification opérationnelle et les politiques hospitalières pour les priorités quant à la disponibilité et l'usage des lits. Les protocoles de surcapacité des hôpitaux, les congés accélérés et les processus de congé plus structurés amélioreront le roulement global des hôpitaux et préviendront l'engorgement des départements d'urgence.

\section{AUTRES SOLUTIONS POSSIBLES}

Plusieurs stratégies ont été utilisées pour résoudre le problème de blocage d'accès et de l'engorgement des départements d'urgence, incluant:

\section{i. Solutions D'AFFLUX}

1. Accès aux soins primaires: Des investissements dans un système rigoureux de soins primaires assurant que tous les Canadiens ont un accès raisonnable à FSP avec une attention axée sur la prévention et la santé. Un accès amélioré et prolongé à un FSP avec un plus grand accès à des rendez-vous semi-urgents et hors des heures normales de travail pourrait possiblement empêcher que les patients tombent malades et par conséquent, ils nécessiteraient moins de soins hospitaliers.

2. Amélioration de la coordination des SMU: Une importance particulière devrait être accordée aux processus de déchargement des SMU. Le recours à des infirmières responsables du déchargement des ambulances en Ontario s'est avéré un succès impressionnant dans l'effort de contrer le problème de blocage d'accès pour les patients pré-hospitalisés. L'Ontario a accordé des fonds spécifiques pour que des infirmières prennent en charge des patients arrivant en ambulance à des périodes de pointe de la journée - même s'il n'y a pas de civières disponibles (des endroits adéquats pour que ceci prennent place se trouvent dans l'aire d'attente/ d'arrivée du DU ou dans un espace adjacent au DU). Les ambulanciers peuvent donc retourner sur la route. L’Alberta a également fait usage des processus de consolidation des SMU pour régler le problème de blocage d'accès des SMU. Dans certains hôpitaux, de nombreux patients des SMU sont consolidés ensemble et un fournisseur de SMU s'occupe d'eux afin de faciliter le retour des équipes d'ambulances dans la communauté.

\section{ii. Solutions D'INTERVENTION}

1. S'engager dans l'amélioration des processus: Des techniques de gestion comme «LEAN» ont démontré que de nombreux processus d'hôpitaux et de DU peuvent être simplifiés et améliorés. ${ }^{19}$

2. Investir dans l'amélioration de la dotation en personnel de nos DU: La plupart des DU sont munis d'effectifs pour répondre aux demandes moyennes de roulement de patients. La hausse d'achalandage à des moments précis de la journée, certaines journées de la semaine et durant des saisons spécifiques est assez prévisible. Une dotation en personnel basé sur le volume de l'achalandage qui assure qu'un nombre adéquat de médecins, d'infirmières, de personnel paraprofessionnel et autres fournisseurs de soins de santé (p. ex: IP, adjoints aux médecins, infirmières formées en gestion des urgences en gériatrie, travailleurs sociaux, physiothérapeutes et ergothérapeutes, planificateurs des congés, etc.) sont présents lorsque leur présence est requise, devrait faire partie du plan de dotation. À noter qu'un volume critique de visites au DU, potentiellement au-dessus de 30 000, est nécessaire pour assurer l'usage efficace de ces ressources supplémentaires.

3. Faire concorder la dotation en personnel à la quantité de patients: De nombreux DU peuvent faire un meilleur travail d'organisation de leurs ressources existantes en analysant les tendances d'arrivées des patients. Des données récentes tirées d'un essai randomisé et contrôlé suggèrent également qu'une modification des quarts de travail peut être étudiée à l'aide de résultats quantitatifs et qualificatifs. ${ }^{20}$ Par ailleurs, l'embauche de personnel pour occuper des fonctions administratives a démontré une augmentation globale de l'efficacité des DU. ${ }^{22}$

4. Intégrer les systèmes d'information sur les DU (SIDU) dans l'infrastructure de base 
des DU: Les SIDU ou les systèmes de suivi des patients, peuvent aider dans la gestion du roulement des patients et de l'usage des ressources. Ces systèmes peuvent également recueillir des données pour aider à prendre des décisions plus éclairées en matière de gestion et pour aider à répondre aux exigences relatives à la déclaration de données. ${ }^{8}$ Les SIDU qui concordent avec nos stratégies et qui intègrent nos définitions et nos cibles peuvent permettre la collecte et la distribution en temps réel de mesures de rendement afin de soutenir la transparence relativement à des perturbations de rendement locales et pour soutenir une meilleure gestion du rendement à tous les niveaux: de l'unité à l'hôpital en passant par la gestion régionale et la gestion de l'ensemble du système.

5. Faire usage des directives médicales: Lorsque combinés avec un programme de processus d'approbation, d'éducation et d'implémentation adéquat, et le suivi en cours, les directives médicales peuvent accélérer les soins pour certains patients à leur arrivée au DU.

6. Faire usage des aires de traitement accéléré: Un grand nombre d'alternatives ont été proposées, telles que la dissuasion du recours au DU par le biais de campagnes médiatiques et le détournement des patients vers des cliniques sans rendez-vous. Toutefois, la plupart des données suggèrent que ces stratégies sont inefficaces. ${ }^{22,23}$ Globalement, malgré le fait que les données soient mal coordonnées, il semblerait qu'il y ait un soutien pour le rôle des aires de traitement accéléré dans la majorité des DU urbains à grand volume. Ces données ne s'apprêtent probablement pas aux hôpitaux plus petits des régions rurales. Plusieurs rapports concluent que l'utilisation d'un système de traitement accéléré dans les DU semble être efficace, rentable sur le plan opérationnel, sécuritaire et improves patient satisfaction with care. ${ }^{7,24}$ L'auteur du rapport le plus exhaustif sur la matière a conclu que: 1 ) les aires de traitement accéléré étaient sécuritaire et ne semblaient pas fournir des soins de moins bonne qualité 2) puisqu'elles nécessitent moins de ressources, les aires de traitement accéléré sont rentables et 3) la qualité de la documentation dans ce secteur est insuffisante..$^{25}$

7. Faire usage de «zones d'évaluation rapide» (ZER): De nombreux DU ont réussi à organiser et à doter en personnel des endroits spécifiques au DU pour répondre à des besoins de populations de patients spécifiques. Les aires de traitement accéléré ou les aires de traitement ambulatoire ou mineur desservent les patients présentant de faibles risques d'hospitalisation et qui n'ont pas besoin de civières. Le personnel peut leur prodiguer des soins dans des endroits non conventionnels et ils nécessitent souvent que d'une civière pendant une très courte durée, soit le temps d'effectuer un examen. S'ensuivent le traitement et le temps d'attente dans des chaises confortables qui prennent moins d'espace au DU et exigent moins de ressources. Les zones d'évaluation rapides ou les ZER peuvent être utilisées pour l'évaluation initiale de patients à gravité intermédiaire qui sont assez stables pour demeurer assis dans une chaise, mais qui requièrent une civière lors de l'évaluation ou lors d'un examen plus intime. ${ }^{26}$

8. Établir des politiques et des processus formalisés en matière de prise en charge des patients: La prise en charge est un processus formalisé qui survient lorsque les patients ont des douleurs ne pouvant pas être évalués au cours de processus de triage et qu'ils peuvent être déplacés vers une zone d'évaluation rapide où un médecin peut effectuer un examen plus formel et rapide et ensuite diriger le patient vers un espace de soin approprié dans le DU. Typiquement, un patient qui présente à titre d'exemple, des douleurs de niveau 3, peut être examiné dans une aire de prise en charge du DU, des investigations peuvent être rapidement initiées et le patient peut ensuite être dirigé vers un espace de soins de courte durée ou vers l'environnement de traitement accéléré/traitement mineur pour compléter leurs soins.

9. Mettre en place des unités de soins de courte durée (USCD), des unités de décisions cliniques (UDC), des unités d'observation (UO) et des unités d'évaluation médicale (UEM): Dans certains 
environnements, l'instauration d'USCD, d'UDC, d'UO et d'UEM bien conçues et surveillées permet une baisse de l'engorgement des départements d'urgence et améliore l'ensemble du roulement des patients et les soins qui leur sont prodigués. D'ordre général, plus le volume de patients et d'hospitalisations dans le DU sont élevés, plus ces types d'unités apportent un effet positif. Selon les récentes données suivant l'implémentation d'USCD en Ontario, les avantages pourraient être moins élevés que les bénéfices publiés auparavant. ${ }^{27}$

10. Faire usage de laboratoires au DU reliés par satellite: En raison des délais associés avec les demandes d'analyse de laboratoires dans les DU qui ont été identifiés dans la littérature médicale, ${ }^{28}$ il est peut-être raisonnable de croire qu'une amélioration de ces délais pourrait avoir une importante influence sur l'ensemble de la DDS. Selon les données résumées par le rapport du Health Quality Council of Alberta (HQCA), l'effet du dépistage aux points de service sur les délais d'exécution est soutenu par des données relativement significatives, tandis que les données soutenant l'effet positif sur la DDU sont plutôt limitées. Globalement, les données suggèrent une réduction de 60 minutes de la durée du séjour grâce à l'utilisation de laboratoires au DU reliés par satellite, bien que les résultats actuels peuvent varier au plan local selon des délais d'exécution de base et les analyses disponibles. ${ }^{6}$

11. Faire usage de meilleures pratiques d'enseignement: L'approche traditionnelle d'enseignement - souvent réalisée avec une évaluation initiale par un interne et une prise de décision ralentie - peut constituer un obstacle au roulement. Nous devons trouver des moyens pour conserver l'expérience d'enseignement tout en maintenant l'attention sur le patient et en préservant l'accès et la qualité.

\section{iii. Solutions d'ISSUE}

1. Instaurer des protocoles de surcapacité: Le blocage d'accès et l'engorgement des départements d'urgence sont le résultat d'une demande supérieure à la capacité des hôpitaux et ces problèmes nécessitent des solutions sur l'ensemble du système. Le blocage d'accès et l'engorgement des départements d'urgence peuvent être résolus immédiatement avec les ressources existantes par le biais de mécanismes visant à améliorer le roulement des patients. L'ACMU recommande l'instauration rapide de protocoles de surcapacité pour que tous les hôpitaux aient une approche organisée pour gérer des situations de demandes supérieures à la capacité de la meilleure manière possible. Bien qu'il y ait peu de données pour soutenir cette intervention, ${ }^{29}$ la mise en place de protocoles de surcapacité partage efficacement la responsabilité de patients déjà stabilisés et hospitalisés sur l'ensemble de l'hôpital, plutôt que de les «entreposer» dans le département d'urgence. Les protocoles de surcapacité devraient être instaurés lors de périodes d'hospitalisation de pointe où les soins prodigués aux patients dans le DU sont compromis. Bien que ces protocoles ne soient pas une solution permanente au blocage d'accès et à l'engorgement des départements d'urgence, ils représentent un mécanisme pour «décompresser» temporairement le DU. Alors que d'autres stratégies mèneront à un meilleur rendement de base des DU, la fréquence d'utilisation de ces protocoles diminuera.

2. Instaurer des politiques et des processus de roulement sur l'ensemble de l'hôpital: Une approche pour améliorer le roulement dans les hôpitaux est de créer un Comité de DDS afin de surveiller et optimiser continuellement le roulement des patients et de minimiser adéquatement la DDS. Ces comités devraient être dirigés par des administrateurs séniors ayant le pouvoir de prise de décisions au niveau local. L'objectif de ces comités serait de contrer de manière agressive les facteurs directement associés à la DDS dans les hôpitaux, tels que:

- La désignation d'un principal médecin responsable (MRP) - ce qui est particulièrement important pour les patients compliqués nécessitant plusieurs services en matière de soins;

- La désignation de planificateurs de congé;

- Les priorités de laboratoire et de radiologie pour les patients hospitalisés; 
- La surveillance et l'amélioration des temps de consultations;

- L'amélioration de la planification de congé au moyen de directives fondées sur des données probantes et des normes;

- L'amélioration de la communication avec les fournisseurs de soins primaires;

- La facilitation des suivis avec les spécialistes;

- L'évaluation des taux de réhospitalisation et l'identification des domaines de préoccupation pour continuer l'amélioration de la qualité;

- La création de cliniques externes et de soins ambulatoires pour promouvoir les congés rapides;

- Une intervention plus rapide des CCAC (soins à domicile).

Les mesures conçues pour aider les hôpitaux à atteindre des cibles de durée de séjour dans les DU doivent être réalisables dans un contexte local. Il n'existe pas de solution universelle. Les problèmes de blocage d'accès et d'engorgement des départements d'urgence doivent être résolus rapidement par une collaboration entre les gouvernements provinciaux, les autorités de la santé, les administrateurs hospitaliers, les organisations d'accès aux soins communautaires, les médecins d'urgence de première ligne et tout le personnel hospitalier afin d'effectuer les changements nécessaires pour offrir un accès sécuritaire aux soins d'urgence et pour améliorer le roulement des patients.

\section{CONCLUSIONS}

Le blocage d'accès et l'engorgement des départements d'urgence représentent une situation d'urgence en santé publique. Les DU bondés sont associés à les résultats négatifs pour les patients, incluant une hausse du taux de mortalité chez les patients hospitalisés lors de périodes de pointe. Le blocage d'accès et l'engorgement des départements d'urgence sont le résultat de problèmes à couches multiples nécessitant un engagement à tous les niveaux des soins de santé. La responsabilité pour les soins des patients s'étend bien au-delà du DU et de l'hôpital, et un accent particulier devrait être mis sur l'accès à des soins de base et la satisfaction des besoins de patients nécessitant un ANS. Les incitations doivent être liées aux comportements désirés, le rendement doit être suivi et rapporté et les cadres dirigeants doivent faire preuve de direction et de responsabilité. Il existe maintenant des exemples nationaux et internationaux de juridictions qui ont réussi à résoudre ce problème avec succès. Il faut agir maintenant; il n'y a plus aucune raison d'attendre.

De plus amples renseignements sur l'engorgement des départements d'urgence et les progrès et les réussites au plan local et provincial peuvent être obtenus par l'Association canadienne des médecins d'urgence au www.caep.ca.

\section{GLOSSAIRE DES TERMES}

ACMU: L'Association canadienne des médecins d'urgence - L'ACMU est le lieu de rencontre pour les médecins d'urgence! La mission de l'ACMU est de promouvoir les intérêts des médecins d'urgence et la spécialité de la médecine d'urgence au Canada en représentant les intérêts des médecins d'urgence et leurs patients, en agissant à titre de liaison pour les médecins d'urgence, en offrant de l'éducation de pointe en médecine d'urgence et un forum pour la recherche en médecine d'urgence.

Adjoint aux médecins - est un professionnel de la santé qui est formé pour exercer la médecine dans le cadre d'une équipe avec un médecin.

ANS: Autre niveau de soins - définit les patients qui ne requièrent plus des soins hospitaliers, mais qui ne peuvent obtenir leur congé en raison du manque de lits ou de ressources. En somme, les patients nécessitant un ANS ne reçoivent pas les bons soins au bon endroit. Ils sont souvent référés comme étant des «bloqueurs de lits», puisqu'ils empêchent les patients requérants des soins de courte durée d'accéder à des lits. Nous devons cependant ne pas oublier qu'ils n'obtiennent pas les soins qu'ils nécessitent non plus.

Blocage d'accès - fait référence à une situation où les patients du département d'urgence (DU) nécessitant des soins d'hospitalisation ne sont pas en mesure d'accéder à des lits d'hôpitaux dans un délai raisonnable, ou à toute situation où les patients nécessitant des soins sont incapables de les obtenir dans un délai raisonnable.

Cible - se réfère à un critère désigné pour des mesures de rendement clés.

Délai de déchargement - est un état lorsqu'une ambulance transporte un patient à l'hôpital et que les ambulanciers doivent demeurer avec le patient jusqu'à ce que le personnel hospitalier le prenne en charge. 
DU: Département d'urgence - un endroit au sein de l'hôpital conçu pour répondre immédiatement aux patients souffrant de problèmes médicaux graves.

EIM: Évaluation initiale du médecin - le premier contact avec un médecin suite à l'arrivée du patient au DU.

Engorgement des départements d'urgence - se définit comme «une situation dans laquelle la demande de services d'urgence excède la capacité d'un département à offrir des soins de qualité dans des délais acceptables».

ÉTG: Échelle canadienne de triage et de gravité pour les départements d'urgence - est un outil permettant aux départements d'urgence (DU) de prioriser de manière uniformisée les besoins des patients à leur arrivée au DU. Les niveaux de l'ÉTG correspondent aux besoins en matière de ressources, incluant le taux d'hospitalisation, mais ils ne sont pas conçus à cette fin et devraient être utilisés avec précaution pour tout objectif autre que le triage des patients.

FSP: Fournisseur de soins primaires - un médecin qui voit des patients à la demande de ceux-ci pour des soins préventifs ou pour des problèmes médicaux communs. Au Canada, cette personne est généralement un médecin de famille. Toutefois, de plus en plus en Amérique du Nord, cette personne peut être une infirmière praticienne, un pédiatre ou un interniste.

Infirmière formée en gestion des urgences en gériatrie - procure une expertise avancée en gérontologie pour le soin de personnes âgées fragiles admis dans le DU qui sont à risque de souffrir d'évènements indésirables, de perte d'indépendance et d'hospitalisation ou de soins de longue durée.

IP: Infirmière praticienne - une infirmière praticienne (IP) est une infirmière possédant un diplôme d'études supérieures en pratique avancée des soins infirmiers.

SIDUC: Système d'information sur les départements d'urgence canadiens - est un groupe de travail qui développe des ressources, des outils et des définitions visant à promouvoir de meilleures collectes et communications de donnés dans les DU.

SIDU: Système d'information du service d'urgence - Un logiciel pour effectuer le suivi de l'arrivée et du départ de patients au DU et pour assister à la gestion du DU.

SMU: Services médicaux d'urgence - les services ambulanciers; un service médical mobile dédié à offrir des soins médicaux de courte durée à l'extérieur de l'hôpital et à effectuer le transport de patients malades ou blessés à un lieu de soins définitifs, évitant ainsi aux patients de se rendre par eux-mêmes à l'hôpital.

SNISA: Système national d'information sur les soins ambulatoires - conserve les données de soins hospitaliers et communautaires d'urgence et ambulatoires (p. ex.: chirurgies d'un jour et cliniques externes).

Soins de longue durée - une installation pouvant offrir une variété de services qui aident à répondre à la fois aux besoins médicaux et non médicaux de personnes souffrant d'une maladie chronique ou d'un handicap et qui ne peuvent prendre soin d'eux-mêmes pendant une période de temps indéterminée (p. ex.: un foyer de soins infirmiers).

UDC: Unité de décisions cliniques - est une unité d'observation située dans le département d'urgence ou dans un lieu adjacent. L'unité est conçue pour fournir à certains patients des capacités de diagnostics et de soins par des médecins et des infirmières, généralement pour une période allant jusqu'à 24 heures, et ce, dans un environnement sécuritaire, efficace et confortable.

UEM: Unité d'évaluation médicale - procure un service pour l'évaluation et le traitement rapides d'une grande portée de conditions médicales. Cette unité améliore l'efficacité du processus d'hospitalisation pour les patients non planifiés en permettant l'évaluation, le soin et le traitement pendant une période désignée (généralement 48 heures) avant le transfert vers l'hôpital ou vers la maison. Le séjour à l'hôpital des patients est éliminé ou réduit considérablement lorsque ce modèle de soins est utilisé.

USCD: Unités de soins de courte durée SSU procure une alternative aux services hospitaliers traditionnels pour les patients ayant de courts séjours à l'hôpital prévus. Voir les UEM, ces concepts se chevauchent.

ZER: Zones d'évaluation rapide - un endroit dans un DU pour faciliter l'efficacité des soins modérés prodigués aux patients. En règle générale, ces patients sont capables d'attendre sur une chaise dans une aire d'attente adjacente aux aires d'examen, mais ils nécessitent une civière pour une évaluation ou un examen plus intime. Ces zones permettent la mise en place d'un lieu intime tout en augmentant la productivité en matière de civières

Remerciements: Les auteurs aimeraient remercier Lee Arbon, membre du personnel de l'ACMU, pour son aide relativement à l'édition de ce document et pour son assistance aux processus de réalisation de ce document et aux membres du Comité des 
affaires publiques de l'ACMU. Les auteurs tiennent également à remercier les Drs Chris Evans et Bruce McLeod pour leur examen approfondi du présent document.

\section{RÉFÉRENCES}

1. Association canadienne des médecins d'urngece et l'Affiliation nationale des infirmières et des infirmers d'urgence. Joint position statement on emergency department overcrowding. 7CMU 2001;3:82-4.

2. Association canadienne des médecins d'urgence. Énoncé de position de l'ACMU: l'engorgement des départements d'urgence 2009. Consulté au www.caep.ca (au mois d'août 2001, 2011).

3. Bond K, Ospina M, Blitz S, et al. Frequency, determinants, and impact of overcrowding in emergency departments in Canada: a national survey of emergency department directors. Healthc Q 2007;10:32-40, doi:10.12927/hcq.2007. 19312.

4. Guttmann A, Schull MJ, Vermeulen MJ, Stukel TA. Association between waiting times and short term mortality and hospital admission after departure from emergency department: population based cohort study from Ontario, Canada. BMF 2011;342:d2983, doi:10.1136/bmj.d2983.

5. Sprivulis PC, Da Silva JA, Jacobs IG, et al. Association between hospital overcrowding and mortality among patients admitted via Western Australian emergency departments. M7A 2006;184:208-12.

6. de Grood JBM, Villa-Roel C, Zwicker K, et al. Overview of interventions to mitigate emergency department overcrowding. Calgary (AB): HQCA; 2013.

7. Rowe BH, Bond K, Ospina MB. et al. Emergency department overcrowding in Canada: what are the issues and what can be done? Ottawa: Agence canadienne des médicaments et des technologies de santé; 2006.

8. Rowe $\mathrm{BH}$, Bond $\mathrm{K}$, Ospina $\mathrm{M}$, et al. Data collection on patients in emergency departments in Canada. 7CMU 2006; 8:417-24.

9. Asplin BR, Magid DJ, Rhodes KV, et al. A conceptual model of emergency department overcrowding. Ann Emerg Med 2003;42:181-4, doi:10.1067/mem.2003.302.

10. Schull MJ, Slaughter PM, Redelmeier DA. Urban emergency department overcrowding: defining the problem and eliminating misconceptions. 7CMU 2002;4:76-83.

11. Fatovich DM, Nagree Y, Sprivulis P. Access block causes emergency department overcrowding and ambulance diversion in Perth, Western Australia. Emerg Med 7 2005;22:3514, doi:10.1136/emj.2004.018002.

12. Han A, Ospina M, Blitz S, et al. Patients presenting to the emergency department: use of other health care services and reasons for presentation. $7 C M U$ 2007;9:428-34.

13. Richardson DB. Increase in patient mortality at 10 days associated with emergency department overcrowding. Med $\mathcal{7}$ Aust 2006;184:213-6.
14. L'Organisation for Economic Co-operation and Development. Statistics. Consulté au www.oecd.org (le 13 mai 2013).

15. Bagust A, Place M, Posnett JW. Dynamics of bed use in accommodating emergency admissions: stochastic simulation model. BMF 1999;319:155-8, doi:10.1136/bmj.319. 7203.155 .

16. Forster AJ, Stiell I, Wells G, et al. The effect of hospital occupancy on emergency department length of stay and patient disposition. Acad Emerg Med 2003;10:127-33, doi:10.1111/j.1553-2712.2003.tb00029.x.

17. Wait Time Alliance, It's about time: achieving benchmarks and best practices in wait time management. Rapport final. 2005.

18. Association canadienne des médecins d'urgence. Document d'information: l'engorgement des départements d'urgence au Canada. 2004.

19. Alberti G. Transforming emergency care in England. October 2004. Available at: 2009;54:504-10, doi:http://aace.org.uk/wpcontent/uploads/2011/11/Transforming-Emergency-Care-inEngland.pdf

20. Dickson EW, Anguelov Z, Vetterick D, et al. Use of LEAN in the emergency department: a case series of 4 hospitals. Ann Emerg Med 2009;54:504-10, doi:10.1016/j.annemergmed. 2009.03.024.

21. Rowe BH V-RC, Lashyn T, Singh M, et al. Emergency department staffing decisions using trial data: what really matters? FCMU 2012;14 suppl 1:S46.

22. Rowe BH, Guo X, Villa-Roel C, et al. The role of triage liaison physicians on mitigating overcrowding in emergency departments: a systematic review. Acad Emerg Med 2011;18: 111-20, doi:10.1111/j.1553-2712.2010.00984.x.

23. Hutchison B, Ostbye T, Barnsley J, et al. Patient satisfaction and quality of care in walk-in clinics, family practices and emergency departments: the Ontario walk-in clinic study Can Med Assoc 7 2003;168:977-83.

24. Affleck A, Innes G. Quality of care in walk-in clinics, family practice and emergency departments: the Ontario walk-in clinic study. FCMU 2003;5:350-2.

25. Yoon P. Emergency department fast-track system. HTA Initiative \#10. Edmonton (AB): Alberta Heritage Foundation for Medical Research; 2003.

26. Bullard MJ, Villa-Roel C, Guo X, et al. The role of a rapid assessment zone/pod on reducing overcrowding in emergency departments: a systematic review. Emerg Med $\mathcal{F} 2012$; 29:372-8, doi:10.1136/emj.2010.103598.

27. Schull MJ, Vermeulen MJ, Stukel TA, et al. Evaluating the effect of clinical decision units on patient flow in seven Canadian emergency departments. Acad Emerg Med 2012;19: 828-36, doi:10.1111/j.1553-2712.2012.01396.x.

28. Yoon P, Steiner I, Reinhardt G. Analysis of factors influencing length of stay in the emergency department. FCMU 2003;5:155-61.

29. Villa-Roel C, Guo X, Holroyd BR, et al. The role of full capacity protocols on mitigating overcrowding EDs. Am 7 Emerg Med 2012;30:412-20, doi:10.1016/j.ajem.2010.12.035. 